\title{
IncRNA:mRNA expression profile in CD4+ T cells from patients with Graves' disease
}

\author{
Qinglei Yin ${ }^{1,2,3, *}$, Zhou Jin ${ }^{1,2, *}$, Yulin Zhou ${ }^{1,2}$, Dalong Song ${ }^{3,4}$, Chenyang Fu ${ }^{1,2}$, Fengjiao Huang ${ }^{4,5}$ and Shu Wang ${ }^{1,2}$ \\ ${ }^{1}$ Department of Endocrine and Metabolic Diseases, Shanghai Institute of Endocrine and Metabolic Diseases, Ruijin Hospital, Shanghai Jiao Tong University \\ School of Medicine, Shanghai, China \\ ${ }^{2}$ Shanghai National Clinical Research Center for Metabolic Diseases, Key Laboratory for Endocrine and Metabolic Diseases of the National Health \\ Commission of the PR China, Shanghai National Center for Translational Medicine, Ruijin Hospital, Shanghai Jiao Tong University School of Medicine, \\ Shanghai, China \\ ${ }^{3}$ Guangdong Geriatric Institute, Guangdong Provincial People's Hospital, Guangdong Academy of Medical Sciences, Guangzhou, China \\ ${ }^{4}$ Reproductive Medicine Center, Guangdong Provincial People's Hospital, Guangdong Academy of Medical Sciences, Guangzhou, China \\ ${ }^{5}$ Division of Endocrinology, Department of Internal Medicine, The First Affiliated Hospital of Zhengzhou University, Zhengzhou, Henan, China
}

Correspondence should be addressed to F Huang or S Wang: huanggua724@126.com or shuwang999@hotmail.com

*(Q Yin and Z Jin contributed equally to this work)

\begin{abstract}
Graves' disease (GD) is a common autoimmune disease that affects the thyroid gland. As a new class of modulators of gene expression, long noncoding RNAs (IncRNAs) have been reported to play a vital role in immune functions and in the development of autoimmunity and autoimmune disease. The aim of this study is to identify IncRNAs in CD4+ T cells as potential biomarkers of GD. IncRNA and mRNA microarrays were performed to identify differentially expressed IncRNAs and mRNAs in GD CD4+ T cells compared with healthy control CD4+ T cells. Quantitative PCR (qPCR) was used to validate the results, and correlation analysis was used to analyze the relationship between these aberrantly expressed IncRNAs and clinical parameters. The microarray identified 164 IncRNAs and 93 mRNAs in GD CD4+ T cells differentially expressed compared to healthy control CD4+ $T$ cells (fold change $>2.0$ and a $P<0.05$ ). Further analysis consistently showed that the expression of HMlincRNA1474 $(P<0.01)$ and TCONS_00012608 $(P<0.01)$ was suppressed, while the expression of $A K 021954(P<0.01)$ and $A B 075506(P<0.01)$ was upregulated from initial GD patients. In addition, their expression levels were recovered in euthyroid GD patients and GD patients in remission. Moreover, these four aberrantly expressed IncRNAs were correlated with GD clinical parameters. Moreover, the areas under the ROC curve were 0.8046, 0.7579, 0.8115 for AK021954, AB075506, HMlincRNA1474, respectively. The present work revealed that differentially expressed IncRNAs were associated with GD, which might serve as novel biomarkers of GD and potential targets for GD treatment.
\end{abstract}
Key Words
- Graves' disease
- CD4+ T cells
- IncRNA
- expression profile

\section{Introduction}

Graves' disease (GD) is an autoimmune disorder characterized by the presence of autoantibodies that bind to and stimulate the thyrotropin receptor, resulting in hyperthyroidism and goiter (1). It is suggested that the environmental factors trigger the occurrence of GD under genetical background, however, the pathogenesis is still incompletely understood (2).
Advances in cellular immunology have opened a new era of or new insights into exploring the mechanisms of immune related diseases. As indispensable components of immunity, CD4+ T lymphocytes play vital roles during the course of GD. The infiltration of T lymphocytes leads to the destruction of thyroid tissues, which synthesize proinflammatory cytokines to maintain and amplify the

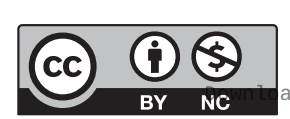

This work is licensed under a Creative Commons Attribution-NonCommercial 4.0 International License. ded from Bioscientifica.com at 04/26/2023 06:38:27AM 
extent the autoimmune response. The imbalance between $\mathrm{T}$ helper (Th) cell 1 and 2 results in GD $(3,4)$. Recent studies have illustrated that other subsets of functional $\mathrm{T}$ cells, such as T follicular helper cells (Tfh) $(5,6)$, Th17 cells $(7,8,9)$, and Th22 cells $(7,9)$, also play an important role in the pathogenesis of GD. Treg cells are characterized by immunosuppressive function, and they play an important role in stabilizing the immune system. Treg cells show altered numbers and defective suppressor function in GD patients $(10,11)$. These findings suggest the dysfunction of CD4+ T cells play an important role in the pathogenesis of GD development. However, the potential mechanisms underlying $\mathrm{CD} 4+\mathrm{T}$ cell dysfunction need to be clarified.

Long noncoding RNAs (IncRNAs) are a large group of noncoding RNAscharacterized bylow conservation, generic or organ specificity, and nucleotide length $>200$ (12). Recently, IncRNAs which have previously been thought to be nonfunctional RNAs, are shown to play important roles in chromatin remodeling, transcription control, post-transcriptional processing, and protein metabolism $(13,14)$. Accumulating studies have shown that lncRNAs are involved in the regulation of autoimmunity- and inflammation-related processes, including nuclear factor$\kappa \mathrm{B}$ and toll-like receptor signaling, cytokine expression, and immune cell proliferation and differentiation $(15,16$, $17,18,19)$. It has also been demonstrated that IncRNAs play an important role in the pathogenesis of a variety of autoimmune diseases, such as multiple sclerosis (20), systemic lupus erythematosus (21), type 1 diabetes and rheumatoid arthritis (RA) (22). However, knowledge of lncRNAs in GD remains limited. In this study, an lncRNA and mRNA expression profile for GD CD4+ T cells were established, and the relationships between the expression levels of aberrantly expressed lncRNAs and clinical indices were analyzed.

\section{Materials and methods}

\section{Patients}

Forty-five initial GD patients, 30 euthyroid GD patients, 12 TRAb negative-conversion GD (GD in remission) patients, and 30 age- and sex-matched healthy control donors (HC) were enrolled from Ruijin Hospital affiliated to Shanghai Jiao Tong University School of Medicine. GD was diagnosed based on clinical symptoms, biochemical indicators of hyperthyroidism and anti-thyrotropin receptor antibody (TRAb) positivity. Euthyroid GD patients were treated with methimazole (MMI) for 2-4 months and reached normal free triiodothyronine $\left(\mathrm{FT}_{3}\right)$ and free thyroxine $\left(\mathrm{FT}_{4}\right)$ levels. GD patients in remission had been treated with MMI for at least 10 months and maintained $\mathrm{FT}_{3}, \mathrm{FT}_{4}$, TSH and TRAb levels in normal range for at least 3 months. Healthy subjects without any past or present history of thyroid disease were enrolled in this study. Clinical parameters, including thyrotropin (TSH), $\mathrm{FT}_{3}, \mathrm{FT}_{4}$, thyroperoxidase antibody (TPOAb), thyroglobulin antibody (TGAb) and TRAb levels, were obtained by routine clinical laboratory methods. The subject characteristics and clinical information are shown in Table 1. All patients gave written informed consent in accordance with the Declaration of Helsinki. The study was approved by the Research Ethics Board of Ruijin Hospital.

\section{CD4+ T cell isolation}

Human PBMCs were obtained from freshly collected blood in heparinized tubes and isolated by Ficoll-Isopaque density gradient centrifugation (Sigma-Aldrich). After centrifugation, the pellet was washed free of platelets and Ficoll. For the purification of CD4+ T cells from fresh PBMCs, positive selection by human CD4 Micro Beads (Miltenyi Biotec, Bergisch Gladbach, Germany) was used

Table 1 Clinical characteristics of healthy controls, initial GD patients, euthyroid GD patients and GD patients in remission.

\begin{tabular}{lcc}
\hline Variable & & Healthy controls \\
\cline { 1 - 1 }$(\mathrm{N} / \mathrm{F})$ & & $30(13 / 17)$ \\
Age (years) & & $35.60 \pm 8.62$ \\
$\mathrm{FT}_{3}(\mathrm{pmol} / \mathrm{L})$ & & $4.39 \pm 0.46$ \\
$\mathrm{FT}_{4}(\mathrm{pmol} / \mathrm{L})$ & $13.08 \pm 1.28$ \\
$\mathrm{TSH}^{\prime}(\mu \mathrm{IU} / \mathrm{mL})$ & & $1.89 \pm 0.80$ \\
$\mathrm{TRAb}(\mathrm{IU} / \mathrm{L})$ & $0.47 \pm 0.18$ \\
$\mathrm{TPOAb}(\mathrm{IU} / \mathrm{mL})$ & & $0.28 \pm 0.24$ \\
$\mathrm{TGAb}(\mathrm{IU} / \mathrm{mL})$ & $2.04 \pm 4.31$ \\
\hline
\end{tabular}

\begin{tabular}{c}
\hline Initial GD \\
\hline $45(15 / 30)$ \\
$39.22 \pm 13.52$ \\
$28.40 \pm 13.24^{\mathrm{a}}$ \\
$41.50 \pm 12.20^{\mathrm{a}}$ \\
$0.002 \pm 0.006^{\mathrm{a}}$ \\
$18.85 \pm 12.04^{\mathrm{a}}$ \\
$312.94 \pm 366.68^{\mathrm{a}}$ \\
$199.32 \pm 312.98^{\mathrm{a}}$
\end{tabular}

\begin{tabular}{c}
\hline Euthyroid GD \\
\hline $30(11 / 19)$ \\
$40.23 \pm 9.69$ \\
$4.40 \pm 0.93^{\mathrm{b} 1}$ \\
$11.3 \pm 2.59^{\mathrm{b} 1}$ \\
$1.60 \pm 2.82^{\mathrm{b} 1}$ \\
$12.23 \pm 10.95^{\mathrm{b} 2}$ \\
$336.39 \pm 388.64$ \\
$144.25 \pm 188.59$
\end{tabular}

\begin{tabular}{c}
\hline GD in remission \\
\hline $12(3 / 9)$ \\
$41.67 \pm 16.13$ \\
$4.27 \pm 0.49^{c}$ \\
$13.59 \pm 2.31^{c}$ \\
$1.76 \pm 1.26^{c}$ \\
$0.97 \pm 0.39^{c}$ \\
$173.54 \pm 251.73$ \\
$60.08 \pm 108.37$
\end{tabular}

\begin{tabular}{c}
\hline Normal range \\
\hline- \\
- \\
$2.63-5.70$ \\
$9.01-19.04$ \\
$0.3500-4.9400$ \\
$<1.75$ \\
$<5.61$ \\
$<4.11$
\end{tabular}

Data are expressed as mean \pm standard deviation according to the distribution.

${ }^{a} p<0.01$, initial GD compared with healthy controls. ${ }^{b 1} p<0.01,{ }^{b 2} p<0.05$, euthyroid GD compared with initial GD. ${ }^{c} p<0.01$, GD in remission compared with initial GD.

$\mathrm{F}$, female; $\mathrm{FT}_{3}$, free triiodothyronine; $\mathrm{FT}_{4}$, free thyroxine; GD, Graves' disease; $\mathrm{M}$, male; $\mathrm{N}$, number; TGAb, thyroglobulin antibody; TPOAb, thyroperoxidase antibody; TRAb, anti-thyrotropin receptor antibody; TSH, thyrotropin 
according to the manufacturer's instructions. The purity of CD4+ T cells was $>95 \%$ as analyzed by flow cytometer (BD Biosciences, Bedford, MA, USA). The isolated CD4+ T cells were used for further research.

\section{RNA isolation and quantitative reverse transcription-polymerase chain reaction (qRT-PCR) analyses}

Total RNA was extracted by TRIzol reagent (Invitrogen) according to the manufacturer's protocols. One microgram of RNA was used for the synthesis of cDNA using reverse transcriptase (TaKaRa) with oligo dT-adaptor primers. Duplicate samples for quantitative PCR were run in an ICycler (ABI, CA, USA). The quantification of the expression of a given gene, expressed as the relative mRNA level compared with that of the control, was calculated with the $2^{-\Delta \Delta \mathrm{Ct}}$ comparative method after normalization to the housekeeping gene Actin. Primer sequences are shown in Supplementary Table 1 (see section on supplementary materials given at the end of this article).

\section{Cell culture and $\mathrm{T}_{3}$ treatment}

Fresh isolated CD4+ T obtained from three healthy subjects were washed with RPMI 1640 twice. Then $5 \times 10^{6}$ cells were cultured on six-well culture plates in a culture medium (RPMI 1640 plus 10\% FBS plus $100 \mathrm{U} / \mathrm{mL}$ penicillin plus $100 \mu \mathrm{g} / \mathrm{mL}$ streptomycin plus $0.5 \mu \mathrm{g} / \mathrm{mL}$ functional antihuman CD3 mAb plus $2 \mu \mathrm{g} / \mathrm{mL}$ functional anti-human CD28 mAb) with or without $\mathrm{T}_{3}$ (Sigma-Aldrich) for $24 \mathrm{~h}$ or 7 days. After $24 \mathrm{~h}$ and 7 days, the cells were harvested, and RNA was extracted for qRT-PCR analysis.

\section{IncRNA microarray}

Three initial GD patients CD4+ T cells and three age- and sex- matched healthy control donors $\mathrm{CD} 4+\mathrm{T}$ cells were sent to Kangchen Biological Services (Shanghai, China). The clinical parameters of these persons were shown in Supplementary Table 2. Total RNA was extracted from six CD4+ T cells samples and hybridized to the gene chip using Human lncRNA 4*180K Array (Arraystar Inc., Rockville, MD, USA) based on the manufacturer's instructions. The arrays were scanned by Agilent Scanner (G2505B; Agilent Technologies Inc.) and acquired raw images were analyzed using Agilent Feature Extraction software (version 10.7). The Gene Spring v11.0 software package offered subsequent data processing. Differentially expressed IncRNAs and mRNAs between the two groups were identified through fold change filtering. Hierarchical clustering and combined analysis were performed using homemade scripts.

\section{Bioinformatics analysis}

Pathway analysis and gene ontology (GO) analysis were applied to explore the potential roles that the differentially expressed mRNAs play in a biological pathway or GO function, including three categories: biological process, cellular component, and molecular function.

\section{IncRNA target prediction}

Identify the targets of differentially expressed lncRNAs via cis- or trans-regulatory effects. Differentially expressed lncRNAs were selected for target predictions. Two independent algorithms were used. The first algorithm searches for target genes acting in cis. With the help of gene annotations at UCSC (http://genome.ucsc.edu/), lncRNAs and potential target genes were paired and visualized using UCSC genome browser. The genes transcribed within $10 \mathrm{kbp}$ window upstream or downstream of lncRNAs were considered as cis-target genes. The second algorithm is based on mRNA sequence complementarity and RNA duplex energy prediction, assessing the impact of lncRNA binding on complete mRNA molecules. Finally, the RNAplex software was used to choose trans-acting target genes (23). RNAplex parameters were set as -e-20.

\section{Statistical analysis}

SPSS software 16.0 and GraphPad Prism 5.0 were used to analyze the data. The two-tailed Student's $t$ test and rank-sum test were used as appropriate to analyze the expression levels between two groups. ANOVA analysis was used for multiple groups. The relationships between the expression levels of lncRNAs and clinical characteristics were analyzed by Person's correlation coefficient. ROC curve and AUC were also established for discriminating GD patients from healthy controls. $P$ values below 0.05 were regarded as statistically significant.

\section{Results}

IncRNA and mRNA expression profile in GD CD4+ T cells

To understand how the IncRNA and mRNA were differentially expressed in GD and $\mathrm{HC} \mathrm{CD} 4+\mathrm{T}$ cells, we performed genome-wide analysis of the expression profiles of lncRNAs and mRNAs from three initial GD patients and

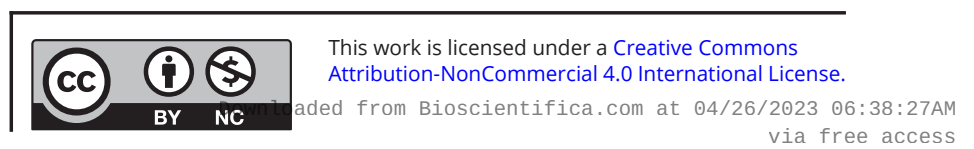


A

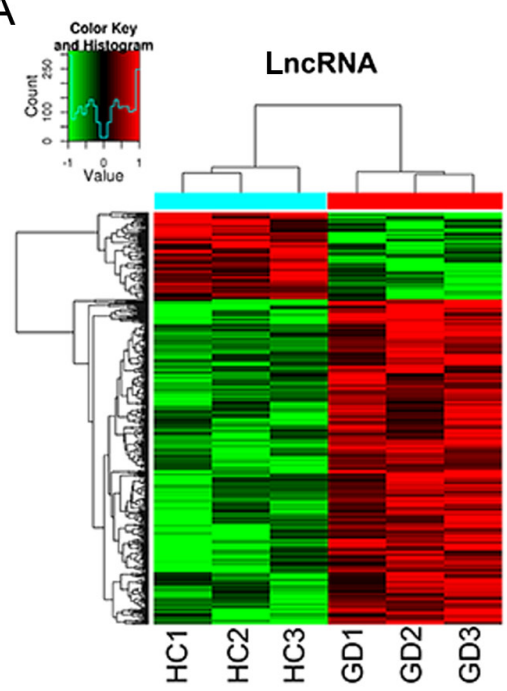

C

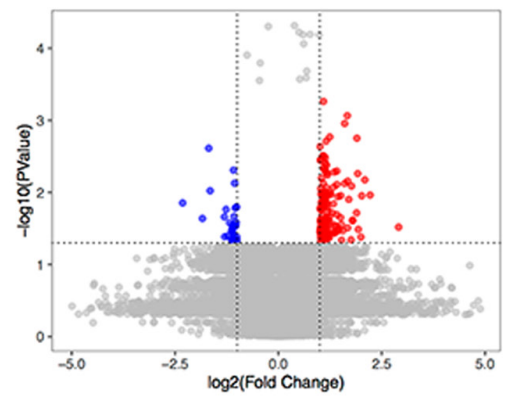

B

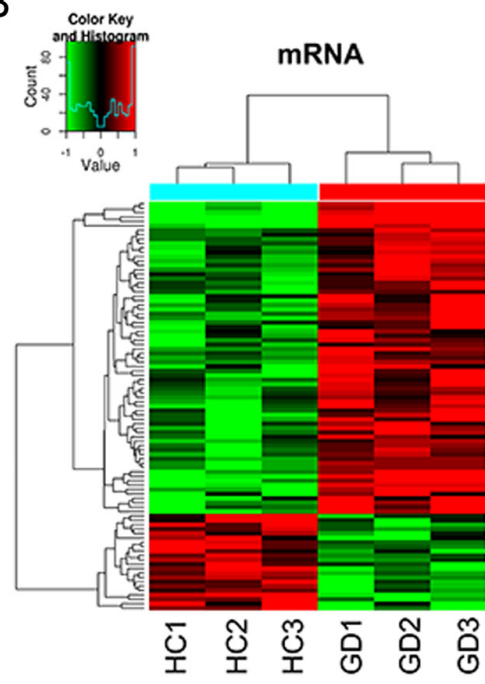

D

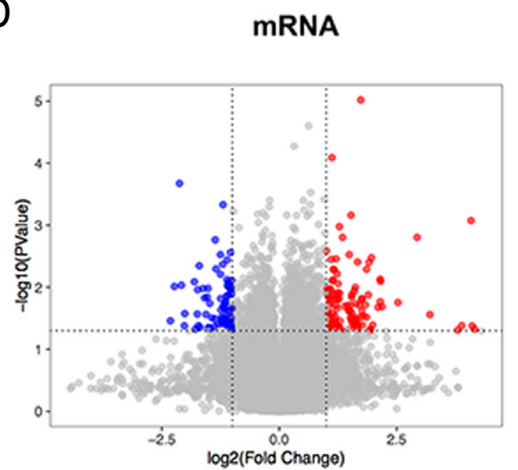

\section{Figure 1}

IncRNA and mRNA expression profile in GD CD4+ $T$ cells. (A) Hierarchical clustering of differentially expressed IncRNAs between GD CD4+ T cells and healthy control CD4+ T cells $(n=3)$. (B) Hierarchical clustering of differentially expressed mRNAs between GD CD4+ T cells and healthy control CD4+T cells $(n=3)$. Each column indicates a different sample. Each row indicates one mRNA or IncRNA. Relatively high expression is indicated by red shading and relatively low expression is indicated by green shading. (C) Volcano plots of IncRNA expression levels between GD CD4+ $T$ cells and healthy control CD4+ T cells $(n=3)$. (D) Volcano plots of mRNA expression levels between GD CD4+ T cells and healthy control CD4+ T cells $(n=3)$. Red squares represent genes upregulated in GD CD4+ T cells and blue squares represent genes downregulated in GD CD4+ $\mathrm{T}$ cells. three healthy controls using Human lncRNA $4{ }^{*} 180 \mathrm{~K}$ Array. Based on the criteria of a fold change $>2.0$ and a $P$ value $<0.05,169$ lncRNAs and 93 mRNAs were differentially expressed between GD and healthy control CD4+ T cells. Of the 169 lncRNAs identified, 29 lncRNAs were downregulated, 140 lncRNAs were upregulated in the GD CD4+ T cells (Supplementary Table 3). Of the 93 mRNAs detected, 71 mRNAs were upregulated and 22 mRNAs were downregulated in GD CD4+ T cells (Supplementary Table 4).

The results of hierarchical cluster analyses showed distinguishable lncRNA and mRNA expression profiles between the healthy control CD4+ T cells and GD CD4+ $\mathrm{T}$ cells (Fig. 1A and B). To visualize the differentially expressed lncRNAs and mRNAs, volcano plot analyses were conducted to further explore the difference (Fig. 1C and D).

\section{GO analysis and pathway analysis}

Gene ontology (GO) and KEGG pathway enrichment analyses were performed to identify the functions of differentially expressed genes. GO project used to classify the significantly regulated genes according to biological process (BP), cellular component (CC), and molecular function (MF). The GO analysis results showed that differentially expressed genes were enriched in cellular process, single-organism process, biological regulation, organelle, binding, catalytic activity and molecular transducer activity (Fig. 2A).

KEGG pathway analysis showed that the differentially expressed genes were enriched for transport and catabolism, signal transduction, infectious diseases and immune system, immune disease, etc. (Fig. 2B and Supplementary Fig. 1).

\section{Validation of differentially expressed IncRNAs}

To validate the results of the microarray analysis, we enrolled 30 healthy volunteers and a total of 87 GD patients, including 45 initial GD patients, 30 euthyroid GD patients and 12 GD in remission patients. All initial GD patients had increased concentrations of $\mathrm{FT}_{3}$ and $\mathrm{FT}_{4}$ and suppressed levels of TSH, whereas after 2-4 months of treatment with anti-thyroid drug MMI, the concentrations of thyroid hormones were recovered to normal, but TRAb level was still relatively high in euthyroid GD. 
A

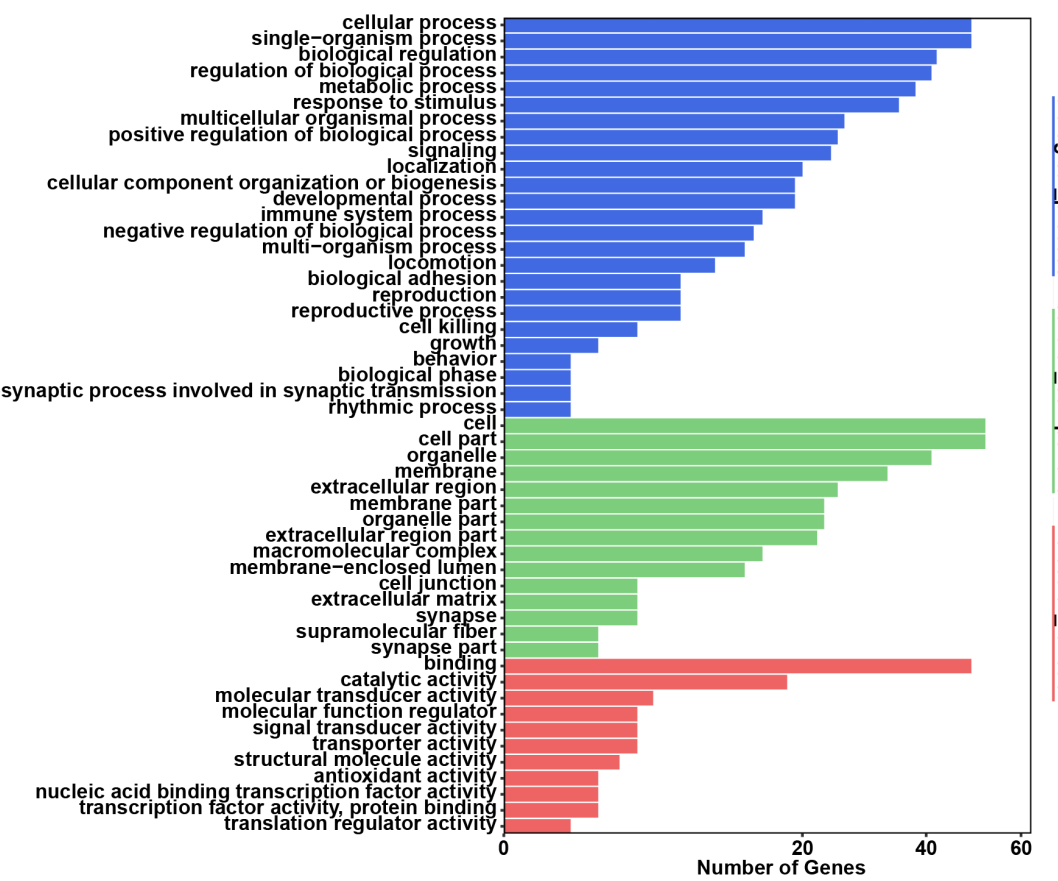

B

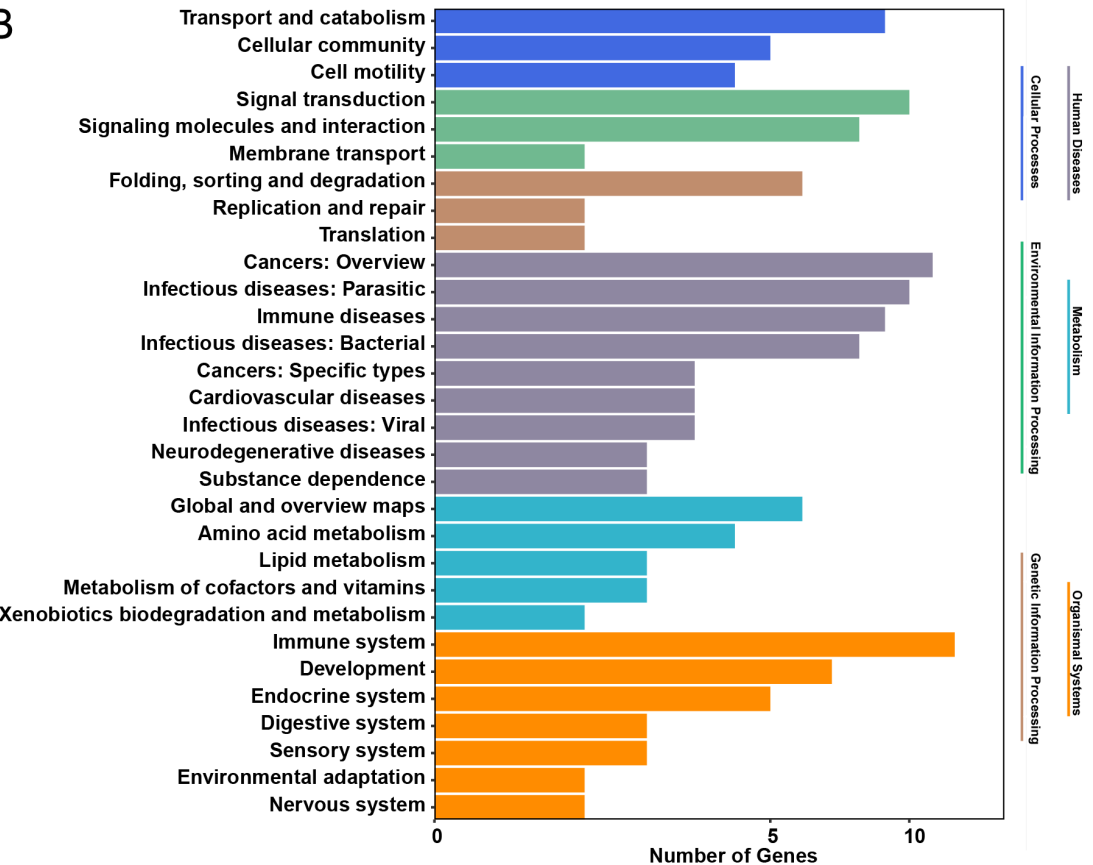

\section{Figure 2}

$\mathrm{GO}$ analysis and pathway analysis. Results of functional enrichment analysis in GD CD4+ T cells compared with HC CD4+ T cells. (A) GO analysis of differentially expressed genes according to biological process, cellular component and molecular function $(n=3)$. (B) Pathway analysis for differentially expressed mRNAs $(n=3)$.
After at least 10 months of treatments, 12 patients obtained normal concentrations of thyroid hormones and TRAb levels, which belong to GD in remission (Table 1).

The most top-ranked upregulated and downregulated lncRNAs were shown in Table 2. Considering the fold changes, the $P$ values, and the specificities of the primers, we chose 15 lncRNAs from Table 2 . Initial GD patients and healthy controls were enrolled for the analysis. The qPCR results confirmed that the expression levels of $A B 075506$,
AL832122, AK055670, AF318328 and AK021954 were increased in the CD4+ T cells of initial GD patients, whereas declined expression of HMlincRNA1474, TCONS_00012608 and AK126108 was observed. They were in consistent with those of the microarray analysis; however, some lncRNAs did not show significant difference between initial GD and HC CD4+ T cells (Fig. 3A).

To further explore the lncRNAs change in the pathogenesis of GD, we assessed the lncRNAs expression

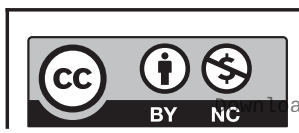

This work is licensed under a Creative Commons Attribution-NonCommercial 4.0 International License. ded from Bioscientifica.com at 04/26/2023 06:38:27AM 
Table 2 The top ten up-regulated and down-regulated IncRNAs in GD group.

\begin{tabular}{|c|c|c|c|}
\hline Sequence name & $P$ value & Fold change & Regulation \\
\hline$A X 721088$ & 0.030 & 7.51 & Up \\
\hline$A B 075506$ & 0.011 & 4.65 & Up \\
\hline ENST00000413452 & 0.007 & 4.26 & Up \\
\hline AL832122 & 0.011 & 4.05 & Up \\
\hline AK056941 & 0.034 & 3.82 & Up \\
\hline HMlincRNA125 & 0.005 & 3.78 & Up \\
\hline AK055670 & 0.002 & 3.73 & Up \\
\hline AF318328 & 0.019 & 3.71 & Up \\
\hline AK021954 & 0.024 & 3.47 & Up \\
\hline AK023574 & 0.024 & 3.46 & Up \\
\hline
\end{tabular}

\begin{tabular}{l}
\hline Sequence name \\
\hline HMlinCRNA1474 \\
TCONS_00012608 \\
TCONS_00014990 \\
ENST00000494340 \\
UC010ggb \\
ENST00000444037 \\
AK058085 \\
AK127903 \\
ENST00000440123 \\
AK126108
\end{tabular}

\begin{tabular}{c}
\hline $\boldsymbol{P}$ value \\
\hline 0.014 \\
0.023 \\
0.002 \\
0.009 \\
0.022 \\
0.041 \\
0.017 \\
0.040 \\
0.026 \\
0.035
\end{tabular}

\begin{tabular}{cc}
\hline Fold change \\
\hline 5.0 \\
3.57 \\
3.23 \\
3.125 \\
2.5 \\
2.44 \\
2.38 \\
2.33 \\
2.27 \\
2.22 \\
\hline
\end{tabular}

\begin{tabular}{l} 
Regulation \\
\hline Down \\
Down \\
Down \\
Down \\
Down \\
Down \\
Down \\
Down \\
Down \\
Down
\end{tabular}

at different stage of GD. Interestingly, in euthyroid group and remission group, the expression of $A K 021954$ (Fig. 3B), AB075506 (Fig. 3C), TCONS_00012608 (Fig. 3D) returned to normal levels, while HMlincRNA1474 (Fig. 3E) did not increase to normal levels.

\section{Association between clinical parameters and aberrantly expressed IncRNAs}

The association between AK021954, AB075506, HMlincRNA1474, TCONS_00012608 and GD clinical parameters $\left(\mathrm{FT}_{3}, \mathrm{FT}_{4}, \mathrm{TSH}, \mathrm{TRAb}, \mathrm{TPOAb}\right.$ and $\left.\mathrm{TGAb}\right)$ was shown in Table 3. The expression level of AK021954 and $A B 075506$ was positively correlated with $\mathrm{FT}_{3}, \mathrm{FT}_{4}$ and $\mathrm{TRAb}$ levels, while negatively associated with TSH. Meanwhile, the expression level of HMlincRNA1474 was negatively correlated with $\mathrm{FT}_{3}, \mathrm{FT}_{4}$ and TRAb levels. Besides, TCONS_00012608 mRNA level was negatively correlated with $\mathrm{FT}_{3}, \mathrm{FT}_{4}$ and $\mathrm{TGAb}$ levels.

To better characterize the relationship between the selective lncRNA and GD as well as evaluate the effect of thyroid hormone on IncRNA regulation in CD4+ T cells, we also examined expression levels of the lncRNA in CD4+ T cells directly under $\mathrm{T}_{3}$ treatment. We isolated fresh CD4+T cells from healthy individuals and cultured them with or without $\mathrm{T}_{3}$ treatment. After both $24 \mathrm{~h}$ and 7 days of cell culture, we found that the expression levels of $A B 075506$, AK021954, HMlincRNA1474 and TCONS_00012608 were not changed (Supplementary Fig. 2). Our results revealed that the change of AB075506, AK021954, HMlincRNA1474 and TCONS_00012608 was not due to thyrotoxicosis.

\section{Evaluation of the selective IncRNAs as biomarkers for the diagnosis of GD}

We generated ROC curves to estimate the sensitivity and specificity for each IncRNA. As shown in Fig. 4, the area under the ROC curve for AK021954, AB075506,

HMlincRNA1474 was $0.8046,0.7579,0.8115$, indicating potential diagnostic value for GD.

\section{Potential targets of the differentially expressed IncRNAs are integrated with differentially expressed mRNAs}

Since lncRNAs regulate the expression of its target genes, the next step is to construct the relationship between the expression profile of the mRNA and predicted target genes of differentially expressed lncRNAs. Procedures for discovering the target genes consisted of two steps. The first step was to predict potential lncRNA targets in the database via target prediction programs. After the first step, we identified 204 mRNAs which could be regulated by lncRNAs via cis-regulation and 313 mRNAs regulated by IncRNAs via trans-regulation. To illustrate the biological function of these target genes, KEGG analysis was performed. The pathway analysis revealed that the target genes were enriched for immune signaling, such as systemic lupus erythematosus, chemokine signaling pathway and pathogenic Escherichia coli infection (Supplementary Fig. 3 ). The second step was to integrate the predicted potential lncRNA targets with the differentially expressed mRNAs in profile, thus an IncRNA and mRNA co-expression was constructed. As illustrated above, AK021954, AB075506 and HMlincRNA1474 expression was associated with TRAb, so we further focused on the co-expression centering on AK021954, AB075506 and HMlincRNA1474, thus JUNB and NRCAM were identified. Consistent with the microarray data, we found that the expression level of JUNB was reduced in initial GD patient CD4+ T cells and significantly recovered in euthyroid GD and GD in remission CD4+ $\mathrm{T}$ cells (Fig. 5A). Besides, the expression level of NRCAM was increased in initial GD patient CD4+ T cells and significantly declined in euthyroid GD and GD in remission CD4+ T cells (Fig. 5B). Furthermore, HMlincRNA1474 was positively correlated with JUNB mRNA expression (Fig. 5C),

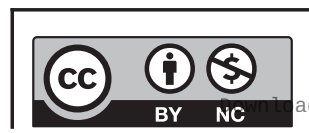

This work is licensed under a Creative Commons Attribution-NonCommercial 4.0 International License. ded from Bioscientifica.com at 04/26/2023 06:38:27AM via free access 
A

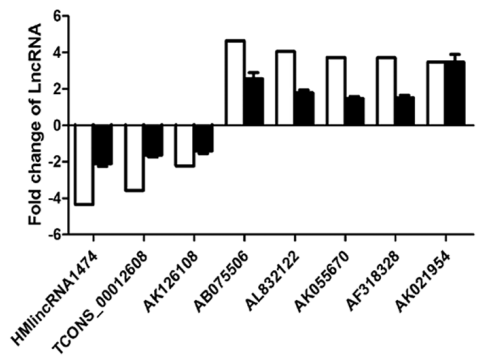

B
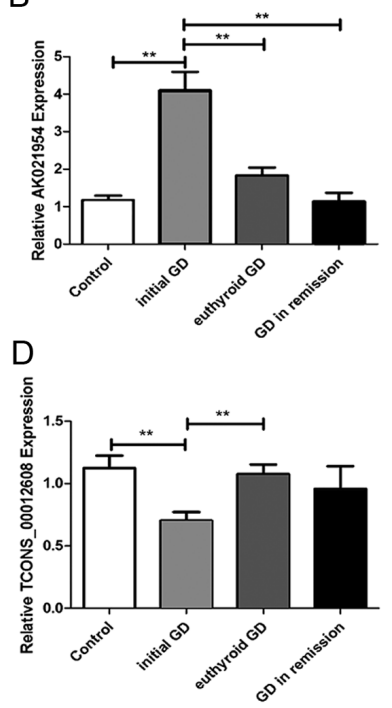

C

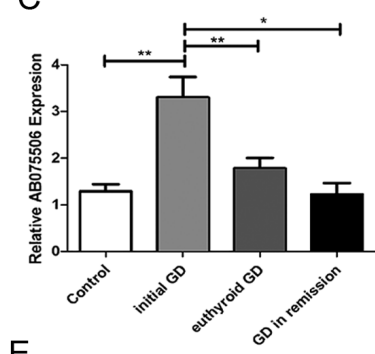

E

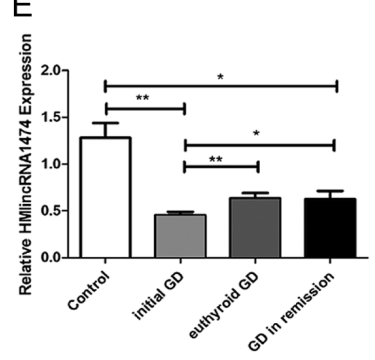

Figure 3

Validation of differentially expressed IncRNAs. (A) The relative expression levels of 8 IncRNAs were validated by qRT-PCR. White, microarray; black, qRT-PCR ( $n=45$ for GD, $n=30$ for HC). (B) Differential AK021954 expression verified by qRT-PCR in CD4+ T cells from healthy controls $(n=30)$, initial GD patients $(n=45)$, euthyroid GD patients $(n=30)$ and GD patients in remission $(n=12)$. (C) Differential $A B 075506$ expression verified by qRT-PCR in CD4+ T cells from healthy controls $(n=30)$, initial GD patients $(n=45)$, euthyroid GD patients $(n=30)$ and GD patients in remission $(n=12)$. (D) Differential TCONS_00012608 expression verified by qRT-PCR in CD4+ T cells from healthy controls ( $n=30)$, initial GD patients $(n=45)$, euthyroid GD patients $(n=30)$ and GD patients in remission $(n=12)$. (E) Differential HMlincRNA1474 expression verified by qRT-PCR in CD4+ T cells from healthy controls $(n=30)$, initial GD patients ( $n=45)$, euthyroid GD patients ( $n=30)$ and GD patients in remission ( $n=12$ ). White bar represents healthy control; light grey bar represents initial GD patients; grey bar represents euthyroid GD patients; black bar represents GD patients in remission. Data represent means \pm S.D. ${ }^{*} P<0.05,{ }^{*} P<0.01$ by Student's $t$ test and one-way ANOVA.

and $A K 021954$ and $A B 075506$ were positively correlated with NRCAM expression (Fig. 5D and E).

\section{Discussion}

The past decade has witnessed an outstanding revolution in the understanding of the regulatory mechanisms controlling gene expression. Accumulating evidence in recent years has shown that noncoding RNAs (ncRNAs) composed of numerous RNA regulatory elements were not directly translated into proteins but were nevertheless capable of exerting a remarkable modulation of gene expression (24, $25,26)$. To date, expression profile studies of cells and tissues have mainly focused on mRNAs and miRNA. With the advancement in the depth and quality of transcriptome sequencing, an increasing number of distinguishably expressed lncRNAs has been illustrated in various disease. Several researches implicated lncRNAs in the development of various diseases, however, there are only scant data related to $\operatorname{GD}(27,28,29)$. In this study, we aimed to explore IncRNA and mRNA expression in GD CD4+T cells to provide new insight into the pathogenesis of GD.

lncRNA-mRNA microarray revealed a set of differentially expressed genes, with 169 differentially expressed lncRNAs and 93 differentially expressed mRNAs in GD CD4+ $\mathrm{T}$ cells when compared with $\mathrm{HC} \mathrm{CD} 4+\mathrm{T}$ cells. Most of these lncRNAs have not been functionally characterized, whereas most of the identified mRNAs are well-known. Therefore, bioinformatics analysis of the aberrantly expressed mRNAs was conducted to help better understand the potential role of $\mathrm{CD} 4+\mathrm{T}$ in the pathological process of GD. GO and pathway analyses showed that differentially expressed mRNAs mainly related to transport and catabolism, signal transduction, infectious diseases and immune system which might be associated with GD pathogenesis. We used qPCR to validate the lncRNA microarray results. Based on the qPCR results, AB075506, AL832122, AK055670, AF318328, AK021954, HMlincRNA1474, TCONS_00012608 and AK126108 were

Table 3 The correlation between the expression levels of IncRNAs and clinical parameters in GD patients.

\begin{tabular}{|c|c|c|}
\hline & \multicolumn{2}{|c|}{$A B 075506$} \\
\hline & $\gamma$ & $P$ \\
\hline FT3 & 0.388 & $<0.001$ \\
\hline FT4 & 0.364 & $<0.001$ \\
\hline TSH & -0.267 & 0.014 \\
\hline TRAb & 0.219 & 0.045 \\
\hline TPOAb & 0.081 & 0.494 \\
\hline TGAb & 0.145 & 0.22 \\
\hline
\end{tabular}

\begin{tabular}{ccc}
\hline \multicolumn{2}{c}{ AK021954 } \\
\hline \multicolumn{1}{c}{$\gamma$} & \multicolumn{1}{c}{$P$} \\
\hline 0.549 & & $<0.001$ \\
0.505 & & $<0.001$ \\
-0.291 & & 0.007 \\
0.366 & & 0.002 \\
0.047 & & 0.687 \\
0.151 & 0.198
\end{tabular}

\begin{tabular}{ccc}
\hline \multicolumn{2}{c}{ HMIincRNA1474 } \\
\hline \multicolumn{1}{c}{$\gamma$} & & $P$ \\
\hline-0.273 & & 0.011 \\
-0.312 & & 0.004 \\
0.195 & & 0.071 \\
-0.216 & & 0.045 \\
-0.068 & & 0.562 \\
0.198 & 0.102 \\
\hline
\end{tabular}

\begin{tabular}{ccc}
\hline \multicolumn{2}{c}{ TCONS_00012608 } \\
\hline$\gamma$ & & $P$ \\
\hline-0.278 & & 0.010 \\
-0.324 & & 0.002 \\
0.107 & & 0.326 \\
-0.156 & & 0.152 \\
-0.074 & & 0.527 \\
-0.231 & & 0.046
\end{tabular}

$\mathrm{FT}_{3}$, free triiodothyronine; $\mathrm{FT}_{4}$, free thyroxine; TGAb, thyroglobulin antibody; TPOAb, thyroperoxidase antibody; TRAb, anti-thyrotropin receptor antibody; $\mathrm{TSH}$, thyrotropin.

https://ec.bioscientifica.com

https://doi.org/10.1530/EC-20-0373 (c) 2020 The authors Published by Bioscientifica Ltd

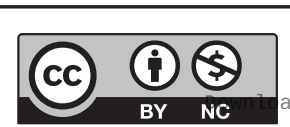

This work is licensed under a Creative Commons Attribution-NonCommercial 4.0 International License. ded from Bioscientifica.com at 04/26/2023 06:38:27AM 

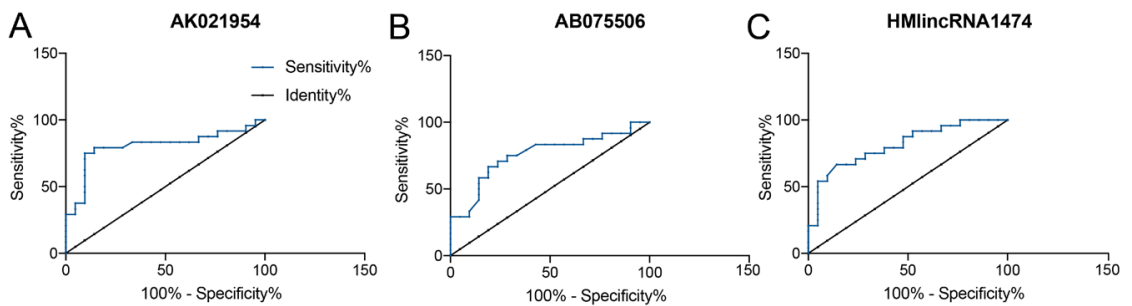

\begin{abstract}
Figure 4
Evaluation of the selective IncRNAs as biomarkers for the diagnosis of GD. The receiver operating characteristic (ROC) curve analysis of $A K 021954$ (A), AB075506 (B), HMlincRNA1474 (C) in the discrimination of Graves' disease (GD) patients from healthy controls.
\end{abstract}

differentially expressed, which was in agreement with the microarray results. Nevertheless, other lncRNAs did not differ significantly in GD CD4+ T cells and healthy control CD4+ T cells on qPCR, in contrast to the results from the microarray. The different trends are likely due to the fact that the expanded test sample size for qRCR might have excluded some of the false positive results obtained in the microarray. Interestingly, AK021954, AB075506, HMlincRNA1424 were correlated with GD clinical parameters, including $\mathrm{FT}_{3}, \mathrm{FT}_{4}$, especially the TRAb levels.

As far as we know, recent studies illustrate that IncRNAs can regulate gene expression either in cis (on neighboring genes) or in trans (distantly located genes) manner $(30,31)$. It is an urgency to understand the mechanisms by which differentially expressed RNAs seek their targets due to the role of lncRNAs in disease development. We used RNAplex algorithms to predict the genes regulated by lncRNAs (32). For the purpose of gaining insight into the function influence of lncRNAs, KEGG pathway annotation was applied to their target gene pool. The analysis showed these genes enriched in immune responses by KEGG annotation, that is, systemic lupus erythematous, chemokine signaling pathway, regulation of actin cytoskeleton and leukocyte transendothelial migration. A lot of researches illustrated that the disruption of proinflammatory cytokines, chemokines and anti-inflammatory cytokine balance played important roles in the pathogenesis of GD (33, $34,35)$. What's more, IncRNAs have been described to contribute to both innate and adaptive immune response $(36,37)$, therefore lncRNAs may involve in GD via inflammatory signaling.

To improve the accuracy of target prediction, we further combined differentially expressed mRNA with target prediction of differentially expressed lncRNAs, and NRCAM and JUNB were identified. Previous reports showed that NRCAM can activate ERK and AKT pathway to inhibit cell apoptosis and promote cell proliferation (38, 39, 40). AKO21954 and AB075506 cis-regulated NRCAM expression in CD4+ T cells, which might increase CD4+ $\mathrm{T}$ cells proliferation and decrease CD4+ T cells apoptosis, thus involving in GD. JUNB is a member of activator

protein 1 (AP-1) (Fos/Jun), a transcriptional regulator of cytokine expression and an important modulator in inflammatory such as rheumatoid, psoriasis and psoriatic arthritis (41). AP-1 has been shown to be involved in the differentiation of naïve $\mathrm{T}$ cells into $\mathrm{T}$ helper 1 cells and $\mathrm{T}$ helper 2 cells, which is a hallmark of the T cell-dependent

A

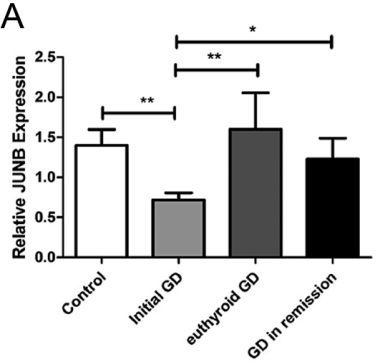

$\mathrm{C}$
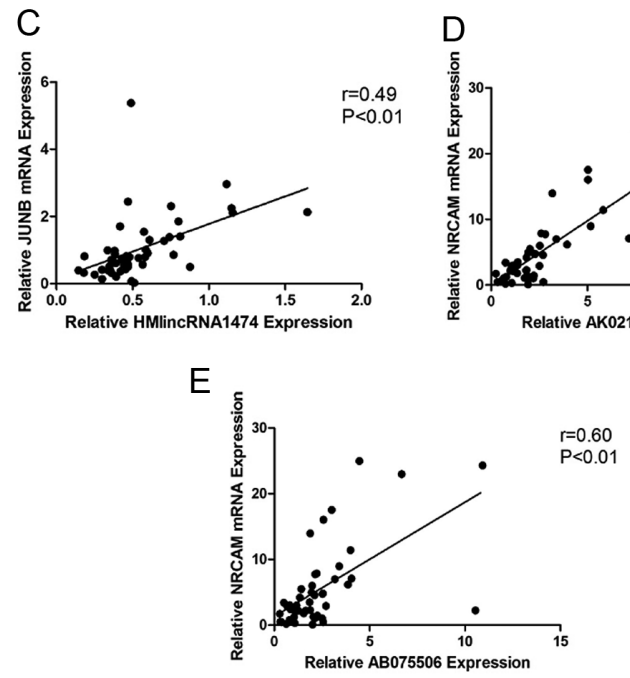

Figure 5

Potential targets of the differentially expressed IncRNAs are integrated with differentially expressed mRNAs. (A) The expression levels of JUNB in initial GD patients $(n=45)$, euthyroid GD patients $(n=30), \mathrm{GD}$ in remission patients $(n=12)$ and healthy controls $(n=30)$ CD4+ T cells by qRT-PCR. (B) The expression levels of NRCAM in initial GD patients $(n=45)$, euthyroid GD patients $(n=30), \mathrm{GD}$ in remission patients $(n=12)$ and healthy controls ( $n=30$ ) CD4+ T cells by qRT-PCR. White bar represents healthy control; light grey bar represents initial GD patients; grey bar represents euthyroid GD patients; black bar represents GD patients in remission. (C) The expression levels of JUNB correlated with HMlincRNA1474. (D) The expression levels of NRCAM correlated with AK0219554. (E) The expression levels of NRCAM correlated with $A B 075506$. Data represent means \pm s.D. $\star P<0.05, * \star P<0.01$ by one-way ANOVA.
This work is licensed under a Creative Commons Attribution-NonCommercial 4.0 International License. ded from Bioscientifica.com at 04/26/2023 06:38:27AM 
immune response. Loss of JUNB in polarized Th2 cells in vitro is followed by deregulated expression of Th2 specific cytokines and by expression of IFN $\gamma$ and T-bet, which are known as key regulators of Th1 cells (42). GD patients harbor activated thyrotropin receptor (TSHR)autoreactive T cells with the dominance of Th1 cytokines $(43,44)$. We propose that decreased JUNB expression in CD4+ $\mathrm{T}$ cells favors Th1 cell differentiation and triggers the autoimmune response.

It is important to acknowledge that there are some limitations in our study. First, the power of the microarray is limited by a relative small sample size, however, the larger sample size of the validation patients has confirmed the microarray results. In addition, the mechanism of these IncRNAs in the pathogenesis of GD is still unknown; further experimental study is still needed.

In summary, our study provided comprehensive IncRNA and mRNA profiles for GD CD4+ $\mathrm{T}$ cells. The differential expression of AK021954, AB075506, HMlincRNA1474 and TCONS_00012608 in GD CD4+ T cells suggested that these IncRNAs may participate in the pathogenesis of GD. Our findings revealed the differentially expressed lncRNAs associated with GD, which might serve as novel biomarkers of GD and potential targets for GD treatment.

\section{Supplementary materials}

This is linked to the online version of the paper at https://doi.org/10.1530/ EC-20-0373.

\section{Declaration of interest}

The authors declare that there is no conflict of interest that could be perceived as prejudicing the impartiality of the research reported.

\section{Funding}

This work was supported by the National Natural Sciences Foundation of China Grants (81873637, 81800699).

\section{Acknowledgements}

The authors would like to thank all the patients and healthy volunteers who have contributed to this study.

\section{References}

1 Smith TJ \& Hegedus L. Graves' disease. New England Journal of Medicine 2016375 1552-1565. (https://doi.org/10.1056/NEJMra1510030)

2 Hemminki K, Li X, Sundquist J \& Sundquist K. The epidemiology of Graves' disease: evidence of a genetic and an environmental contribution. Journal of Autoimmunity 201034 J307-J313. (https:// doi.org/10.1016/j.jaut.2009.11.019)

3 Lichiardopol C \& Mota M. The thyroid and autoimmunity. Romanian Journal of Internal Medicine 200947 207-215.
4 Fallahi P, Ferrari SM, Ragusa F, Ruffilli I, Elia G, Paparo SR \& Antonelli A. Th1 chemokines in autoimmune endocrine disorders. Journal of Clinical Endocrinology and Metabolism 2020105 dgz289. (https://doi.org/10.1210/clinem/dgz289)

5 Zhu C, Ma J, Liu Y, Tong J, Tian J, Chen J, Tang X, Xu H, Lu L \& Wang S. Increased frequency of follicular helper T cells in patients with autoimmune thyroid disease. Journal of Clinical Endocrinology and Metabolism 201297 943-950. (https://doi.org/10.1210/jc.20112003)

6 Liu Y, Yuan X, Li X, Cui D \& Xie J. Constitutive changes in circulating follicular helper $\mathrm{T}$ cells and their subsets in patients with Graves' disease. Journal of Immunology Research 201820188972572. (https://doi.org/10.1155/2018/8972572)

7 Peng D, Xu B, Wang Y, Guo H \& Jiang Y. A high frequency of circulating th22 and th17 cells in patients with new onset Graves' disease. PLoS ONE 20138 e68446. (https://doi.org/10.1371/journal.pone.0068446)

8 Kunisato T, Watanabe M, Inoue N, Okada A, Nanba T, Kobayashi W, Inoue Y, Katsumata Y, Omori N, Nobuhara T, et al. Polymorphisms in Th17-related genes and the pathogenesis of autoimmune thyroid disease. Autoimmunity 201851 360-369. (https://doi.org/10.1080/08 916934.2018.1534963)

9 Noyola M, Ramos-Levi AM, Martinez-Hernandez R, SerranoSomavilla A, Sampedro-Nunez M, Gonzalez-Amaro R \& Marazuela M. Pathogenic Th17 and Th22 cells are increased in patients with autoimmune thyroid disorders. Endocrine 201757 409-417. (https:// doi.org/10.1007/s12020-017-1361-y)

10 Mao C, Wang S, Xiao Y, Xu J, Jiang Q, Jin M, Jiang X, Guo H, Ning G \& Zhang Y. Impairment of regulatory capacity of CD4+CD25+ regulatory T cells mediated by dendritic cell polarization and hyperthyroidism in Graves' disease. Journal of Immunology 2011186 4734-4743. (https://doi.org/10.4049/ jimmunol.0904135)

11 Chen Z, Wang Y, Ding X, Zhang M, He M, Zhao Y, Hu S, Zhao F, Wang J, Xie B, et al. The proportion of peripheral blood Tregs among the CD4+ T cells of autoimmune thyroid disease patients: a meta-analysis. Endocrine Journal 202067 317-326. (https://doi. org/10.1507/endocrj.EJ19-0307)

12 Uchida S \& Jones SP. RNA editing: unexplored opportunities in the cardiovascular system. Circulation Research 2018122 399-401. (https://doi.org/10.1161/CIRCRESAHA.117.312512)

13 Mercer TR, Dinger ME \& Mattick JS. Long non-coding RNAs: insights into functions. Nature Reviews: Genetics 200910 155-159. (https:// doi.org/10.1038/nrg2521)

14 St Laurent G, Wahlestedt C \& Kapranov P. The Landscape of long noncoding RNA classification. Trends in Genetics 201531 239-251. (https://doi.org/10.1016/j.tig.2015.03.007)

15 Aune TM, Crooke PS 3rd, Patrick AE, Tossberg JT, Olsen NJ \& Spurlock CF 3rd. Expression of long non-coding RNAs in autoimmunity and linkage to enhancer function and autoimmune disease risk genetic variants. Journal of Autoimmunity 2017 81 99-109. (https://doi.org/10.1016/j.jaut.2017.03.014)

16 Xia M, Liu J, Liu S, Chen K, Lin H, Jiang M, Xu X, Xue Y, Liu W, Gu Y, et al. Ash1l and lnc-Smad3 coordinate Smad3 locus accessibility to modulate iTreg polarization and T cell autoimmunity. Nature Communications 20178 15818. (https://doi.org/10.1038/ ncomms15818)

17 Zemmour D, Pratama A, Loughhead SM, Mathis D \& Benoist C. Flicr, a long noncoding RNA, modulates Foxp3 expression and autoimmunity. PNAS 2017114 E3472-E3480. (https://doi. org/10.1073/pnas.1700946114)

18 Liu ML, Williams KJ \& Werth VP. Microvesicles in autoimmune diseases. Advances in Clinical Chemistry 201677 125-175. (https:// doi.org/10.1016/bs.acc.2016.06.005)

19 Wang J, Peng H, Tian J, Ma J, Tang X, Rui K, Tian X, Wang Y, Chen J, $\mathrm{Lu} \mathrm{L}$, et al. Upregulation of long noncoding RNA TMEVPG1 enhances T helper type 1 cell response in patients with Sjögren syndrome.

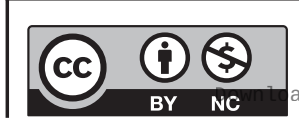

This work is licensed under a Creative Commons Attribution-NonCommercial 4.0 International License. ded from Bioscientifica.com at 04/26/2023 06:38:27AM 
Immunologic Research 201664 489-496. (https://doi.org/10.1007/ s12026-015-8715-4)

20 Zhang F, Gao C, Ma XF, Peng XL, Zhang RX, Kong DX, Simard AR \& Hao JW. Expression profile of long noncoding RNAs in peripheral blood mononuclear cells from multiple sclerosis patients. CNS Neuroscience and Therapeutics 201622 298-305. (https://doi.org/10.1111/cns.12498)

21 Wu Y, Zhang F, Ma J, Zhang X, Wu L, Qu B, Xia S, Chen S, Tang Y \& Shen N. Association of large intergenic noncoding RNA expression with disease activity and organ damage in systemic lupus erythematosus. Arthritis Research and Therapy 201517 131. (https:// doi.org/10.1186/s13075-015-0632-3)

22 Li Z, Li X, Jiang C, Qian W, Tse G, Chan MTV \& Wu WKK. Long non-coding RNAs in rheumatoid arthritis. Cell Proliferation 201851 e12404. (https://doi.org/10.1111/cpr.12404)

23 Tafer H \& Hofacker IL. RNAplex: a fast tool for RNA-RNA interaction search. Bioinformatics 200824 2657-2663. (https://doi.org/10.1093/ bioinformatics/btn193)

24 Khalil AM, Guttman M, Huarte M, Garber M, Raj A, Rivea Morales D, Thomas K, Presser A, Bernstein BE, van Oudenaarden A, et al. Many human large intergenic noncoding RNAs associate with chromatin-modifying complexes and affect gene expression. PNAS 2009106 11667-11672. (https://doi.org/10.1073/pnas.0904715106)

25 Guttman M, Amit I, Garber M, French C, Lin MF, Feldser D, Huarte M, Zuk O, Carey BW, Cassady JP, et al. Chromatin signature reveals over a thousand highly conserved large non-coding RNAs in mammals. Nature 2009458 223-227. (https://doi.org/10.1038/nature07672)

26 Mattick JS \& Makunin IV. Non-coding RNA. Human Molecular Genetics 200615 R17-R29. (https://doi.org/10.1093/hmg/ddl046)

27 Jiang X, Wang Y, Li X, He L, Yang Q, Wang W, Liu J \& Zha B. Microarray profile of B cells from Graves' disease patients reveals biomarkers of proliferation. Endocrine Connections 20209 405-417. (https://doi.org/10.1530/EC-20-0045)

28 Christensen NJ, Gurli H \& Palle B. Decrease in TSH receptor autoantibodies during antithyroid treatment: relationship with a long noncoding Heg RNA and Cdk1 mRNA in mononuclear cells. International Scholarly Research Network Endocrinology 20112011 287052. (http://doi.org/10.5402/2011/287052)

29 Yin L, Zeng C, Yao J \& Shen J. Emerging roles for noncoding RNAs in autoimmune thyroid disease. Endocrinology 2020161 bqaa053. (https://doi.org/10.1210/endocr/bqaa053)

30 Hung T \& Chang HY. Long noncoding RNA in genome regulation: prospects and mechanisms. RNA Biology 20107 582-585. (https:// doi.org/10.4161/rna.7.5.13216)

31 Qureshi IA, Mattick JS \& Mehler MF. Long non-coding RNAs in nervous system function and disease. Brain Research 20101338 20-35. (https://doi.org/ 10.10 16/j. brain res.2 010.03 .110 )

32 Han L, Zhang K, Shi Z, Zhang J, Zhu J, Zhu S, Zhang A, Jia Z, Wang G, Yu S, et al. LncRNA profile of glioblastoma reveals the potential role of lncRNAs in contributing to glioblastoma pathogenesis. International Journal of Oncology 201240 2004-2012. (https://doi.org/10.3892/ijo.2012.1413)

$33 \mathrm{Li} \mathrm{H} \&$ Wang T. The autoimmunity in Graves's disease. Frontiers in Bioscience 201318 782-787. (https://doi.org/10.2741/4141)

34 Yao Q, Wang B, Jia X, Li Q, Yao W \& Zhang J. Increased human interleukin-34. expression is related to disease activity of Graves' disease. Frontiers in Endocrinology 201910 613. (https://doi. org/10.3389/fendo.2019.00613)

35 Yao QM, Li L, Song ZY, Wang B, Qin Q, An XF \& Zhang JA. Elevated interleukin-36 $\alpha$ and CD4(+)IL-36 $\alpha(+)$ T cells are involved in the pathogenesis of Graves' disease. Frontiers in Endocrinology 20189591. (https://doi.org/10.3389/fendo.2018.00591)

36 Schulte LN, Bertrams W, Stielow C \& Schmeck B. ncRNAs in inflammatory and infectious diseases. Methods in Molecular Biology 20191912 3-32. (https://doi.org/10.1007/978-1-4939-8982-9_1)

$37 \mathrm{Xu} \mathrm{J} \&$ Cao X. Long noncoding RNAs in the metabolic control of inflammation and immune disorders. Cellular and Molecular Immunology 201916 1-5. (https://doi.org/10.1038/s41423-0180042-y)

38 Dhodapkar KM, Friedlander D, Scholes J \& Grumet M. Differential expression of the cell-adhesion molecule Nr-CAM in hyperplastic and neoplastic human pancreatic tissue. Human Pathology 200132 396-400. (https://doi.org/10.1053/hupa.2001.23526)

39 Sehgal A, Boynton AL, Young RF, Vermeulen SS, Yonemura KS, Kohler EP, Aldape HC, Simrell CR \& Murphy GP. Cell adhesion molecule Nr-CAM is over-expressed in human brain tumors. International Journal of Cancer 199876 451-458. (https://doi. org/10.1002/(sici)1097-0215(19980518)76:4<451::aid-ijc1>3.0.co;2-q)

40 Conacci-Sorrell ME, Ben-Yedidia T, Shtutman M, Feinstein E, Einat P $\&$ Ze'ev AB. Nr-CAM is a target gene of the beta-catenin/LEF-1 pathway in melanoma and colon cancer and its expression enhances motility and confers tumorigenesis. Genes and Development 200216 2058-2072. (https://doi.org/10.1101/gad.227502)

41 Zenz R, Eferl R, Scheinecker C, Redlich K, Smolen J, Schonthaler HB, Kenner L, Tschachler E \& Wagner EF. Activator protein 1 (Fos/Jun) functions in inflammatory bone and skin disease. Arthritis Research and Therapy 200810 201. (https://doi.org/10.1186/ar2338)

42 Hartenstein B, Teurich S, Hess J, Schenkel J, Schorpp-Kistner M \& Angel P. Th2 cell-specific cytokine expression and allergen-induced airway inflammation depend on JunB. EMBO Journal 200221 6321-6329. (https://doi.org/10.1093/emboj/cdf648)

43 Rapoport B \& McLachlan SM. Graves' hyperthyroidism is antibodymediated but is predominantly a Th1-type cytokine disease. Journal of Clinical Endocrinology and Metabolism 201499 4060-4061. (https:// doi.org/10.1210/jc.2014-3011)

44 Fallahi P, Ferrari SM, Ragusa F, Ruffilli I, Elia G, Paparo SR \& Antonelli A. Th1 chemokines in autoimmune endocrine disorders. Journal of Clinical Endocrinology and Metabolism 2020105 dgz289. (https://doi.org/10.1210/clinem/dgz289)

Received in final form 29 September 2020

Accepted 13 October 2020

Accepted Manuscript published online 13 October 2020 https://ec.bioscientifica.com https://doi.org/10.1530/EC-20-0373 (c) 2020 The authors Published by Bioscientifica Ltd

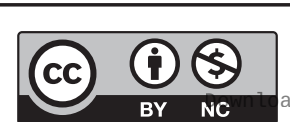

This work is licensed under a Creative Commons Attribution-NonCommercial 4.0 International License. ded from Bioscientifica.com at 04/26/2023 06:38:27AM 